\title{
High-Speed Direct-Detection Electron Detector for the TEAM Project
}

\author{
P. Denes, ${ }^{*}$ M. Battaglia, ${ }^{* *}$ D. Contarato, ${ }^{*}$ D. Doering, ${ }^{*}$ P. Giubilato***, D. Gnani*, B. Krieger* \\ and V. Radmilovic* \\ * Lawrence Berkeley National Laboratory, Berkeley, CA 94720, \\ ** Department of Physics, University of California Santa Cruz, CA 95064 \\ *** Dipartimento di Fisica, Universita` degli Studi, Padova, I-35131, Italy
}

Monolithic CMOS Active Pixel Sensors (APS), first described in 1967 [1],[2] are widely used today in both low- and high-end digital photography. The same technology can, with suitable modification, be used as a direct detector of electrons [3],[4]. For the TEAM (Transmission Electron Aberration-corrected Microscope) project [5]. a 400 frame-per-second APS-based detector has been developed. This detector was engineered through a sequence of design optimizations including precise modeling of electron interactions in the detector together with radiation hardening [6],[7] together with improvements in imaging modes [8]. The detector has a pixel pitch of $9.5 \mu \mathrm{m}$, with a Point Spread Function (PSF) of roughly $8 \mu \mathrm{m}$ at $300 \mathrm{keV}$. In a "cluster counting" mode, the PSF can be reduced to $2 \mu \mathrm{m}$, as shown in FIG. 1 .

The operation of the TEAM detector, together with initial experimental results, will be described. Current developments, in technology further advanced than that used for TEAM will also be described.

\section{References}

[1] G. Wecklers, ."Operation of p-n junction photodetectors in a photon flux integrating mode"., IEEE J. Solid-State Circuits, Vol. SC-2, p. 65, 1967.

[2] P. Noble, ."Self-scanned image detector arrays"., IEEE Trans. Electron Devices, Vol. ED-15, p. 202, 1968.

[3] NH Xuong, et al. "First use of a high-sensitivity active pixel sensor array as a detector for electron microscopy", Proc. SPIE, Vol. 5301 (2004) 242.

[4] A.R. Faruqi, D. M. Cattermole and C. Raeburn, Nucl.Instrum.Meth., Vol. 513 (2003) 317.

[5] C. Kisielowski, et al. "Detection of Single Atoms and Buried Defects in Three Dimensions by Aberration-corrected Electron Microscopy with $0.5 \AA$ Information Limit”,, Microscopy and Microanalysis 14454-462.

[6] M. Battaglia et al., "A Rad-hard CMOS Active Pixel Sensor for Electron Microscopy”, Nucl.Instrum.Meth., Vol. 598 (2009) 642.

[7] M. Battaglia et al., CMOS Pixel Sensor Response to Low Energy Electrons in Transmission Electron Microscopy", Nucl.Instrum.Meth., Vol.605 (2009) 350.

[8] M. Battaglia et al., "Cluster Imaging with a Direct Detection CMOS Pixel Sensor in Transmission Electron Microscopy”, Nucl.Instrum.Meth., Vol.608 (2009) 363. 


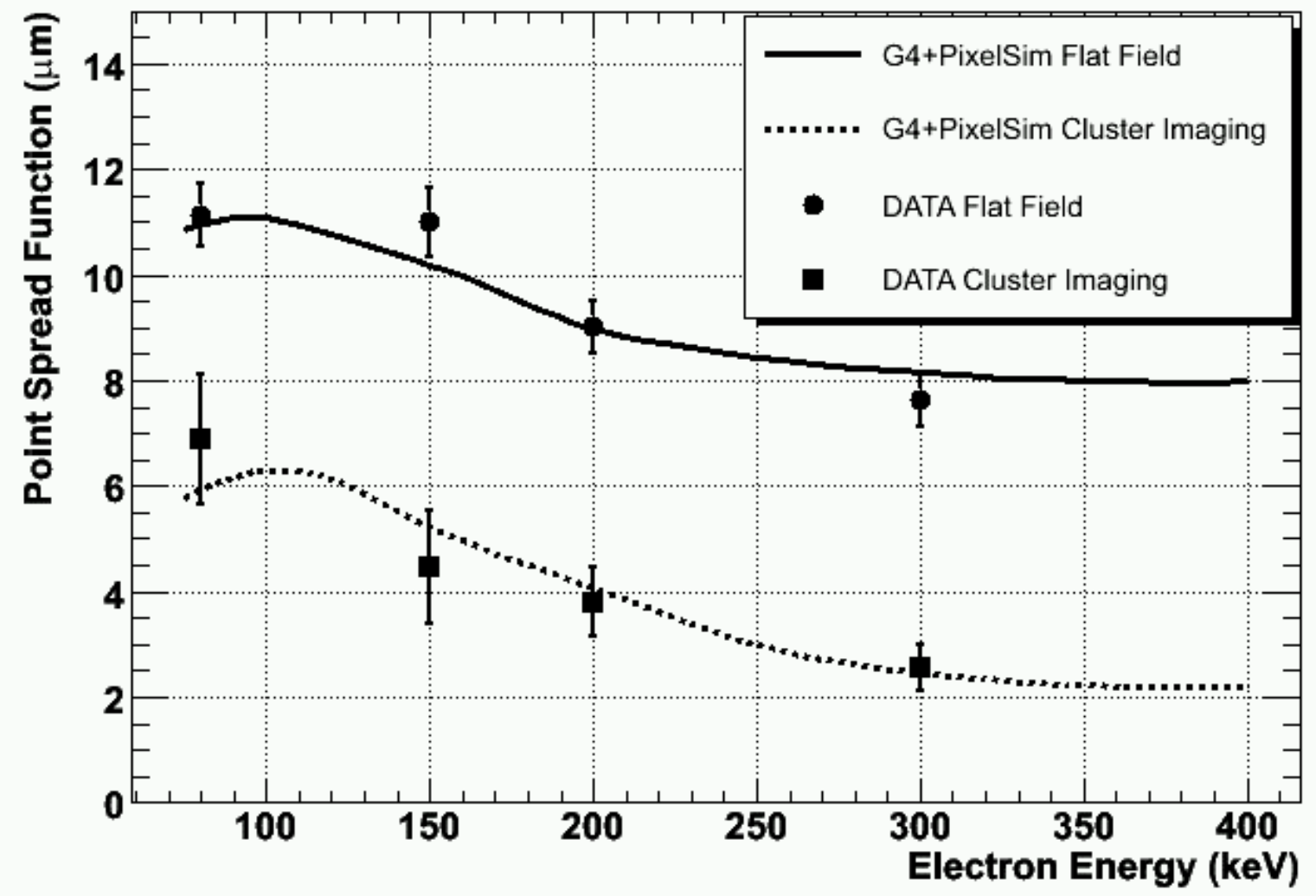

FIG. 1. Point Spread Function in the TEAM detector as a function of electron energy. Points represent measured values in "integrating" and "counting" imaging modes, and curves represent predictions from simulation. 